\title{
Comparison of Inter- and Intra-Individual Neuromuscular Patterns of Responses During Moderate-Load Bilateral Leg Extension Exercise Original Research
}

Taylor K. Dinyer-McNeely ${ }^{1}$, Pasquale J. Succi ${ }^{2}$, Caleb C. Voskuil ${ }^{3}$, M. Travis Byrd ${ }^{4}$, and Haley C. Bergstrom $^{2}$

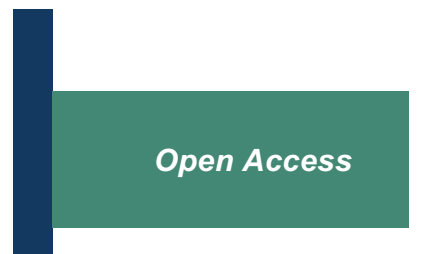

Published: August 26, 2021

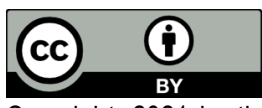

Copyright, 2021 by the authors. Published by Research Directs and the work is licensed under the Creative Commons Attribution $4.0 \quad$ International License. To view a copy of this license, visit http://creativecommons .org/licenses/by/4.0/

Research Directs in Strength and

Performance: 2021,

Volume 1 (Issue 1): 7

ISSN: 2768-5187
${ }^{1}$ School of Kinesiology, Applied Health and Recreation, Oklahoma State University, Stillwater, OK, USA

${ }^{2}$ Department of Kinesiology and Health Promotion, University of Kentucky, Lexington, KY, US A

${ }^{3}$ Department of Kinesiology, Texas Christian University, Fort Worth, TX, USA

${ }^{4}$ Department of Cardiovascular Disease, Mayo Clinic, Scottsdale, AZ, USA

\begin{abstract}
Introduction: This study examined the electromyographic (EMG) and mechanomyographic (MMG), amplitude (AMP) and mean power frequency (MPF) responses during bilateral, leg extension exercise performed to failure at a moderate (70\% one-repetition maximum [1RM]) load.

Methods: Eleven men completed a 1RM and repetitions to failure at $70 \% 1 \mathrm{RM}$ of the leg extension. The EMG and MMG signals were recorded from the right and left vastus lateralis. Polynomial regression analyses were used to determine individual and composite, normalized neuromuscular responses for both limbs.

Results: For EMG AMP, both limbs demonstrated positive, quadratic relationships. For EMG MPF, the right limb demonstrated a negative, cubic relationship and the left limb demonstrated a negative, quadratic relationship. For MMG AMP, the right limb demonstrated a positive, quadratic relationship and the left limb demonstrated a positive, linear relationship. For MMG MPF, both limbs demonstrated negative, linear relationships. $18-45 \%$ of the subjects demonstrated the same responses as the composite for the EMG and MMG signals. $14 \%$ of the subjects demonstrated the same direction and pattern of response for the right and left limb intra-individual responses.

Conclusions: Examining the inter- and intra-individual responses may help identify potential incongruencies in the fatigue response between limbs and potential limb imbalances.
\end{abstract}

Key Words: electromyography, mechanomyography, limb symmetry

Corresponding author: Taylor K. Dinyer-McNeely, taylor.dinyer@okstate.edu

\section{Introduction}

Electromyography (EMG) and mechanomyography (MMG) measure the electrical and mechanical components of muscle, ${ }^{1,2}$ respectively, and can be used to examine the fatigue response during repetitive muscle actions. Both have time (amplitude [AMP]) and frequency (mean power frequency [MPF]) domains that reflect different components of a muscle contraction..$^{1,2,3,4}$ For EMG, the AMP reflects muscle excitation and includes both motor unit recruitment and motor unit firing rate, and the MPF reflects the motor unit action potential conduction velocity traveling along the sarcolemma. ${ }^{1,4}$ The MMG signal records the lateral oscillations of the muscle 
fibers such that the AMP reflects motor unit recruitment and the MPF reflects motor unit firing rate. . $^{2,3}$ Together, the EMG and MMG signals can provide insight on the motor unit activation strategy used to maintain force production during fatiguing tasks. Previously, investigators have used the EMG and MMG signals to examine fatigue-induced changes in the neuromuscular responses throughout a fatiguing task. Typically, investigators have reported increases in EMG and MMG AMP and decreases in EMG and MMG MPF over time during fatiguing isokinetic, isometric, and dynamic constant external resistance (DCER) muscle actions. ${ }^{5,6,7,8}$ These responses indicate there are increases in muscle excitation and motor unit recruitment and decreases in the motor unit action potential conduction velocity and the motor unit firing rate to maintain force production during the fatiguing task.

More recently, investigators have examined the neuromuscular responses of the right and left limbs to compare the inter- and intra-individual and composite responses during fatiguing bilateral muscle actions..$^{5,6,8,9}$ During the performance of maximal, bilateral, isokinetic muscle actions of the leg extensors performed at $180^{\circ} \mathrm{s}^{-1}$, authors reported no difference in the time course or onset of fatigue-induced changes in neuromuscular responses (EMG and MMG, AMP and MFP) between the right and left limbs.,5,9 When examining the composite (i.e., mean of all subjects) response for EMG and MMG, AMP and MPF for both right and left limbs, investigators have reported consistency in the direction (i.e., positive or negative) of fatigue-induced changes in the neuromuscular responses between right and left limbs, as well as the same pattern (i.e., linear, quadratic, or no relationship) and direction for $25 \%,{ }^{9} 50 \%,{ }^{8}$ and $75 \%,{ }^{6,8}$ of the signals in the composite data. Further, a range from $0 \%$ to $92 \%$ of individual subjects demonstrated the same pattern and direction of response for the EMG and MMG signals as the composite response for the right and left limbs. ${ }^{6,8,9}$ This indicates a wide range in variability and consistency between the individual and composite neuromuscular responses during fatiguing bilateral muscle actions of the leg extensors that may suggest the composite response does not always portray the majority response of individual subjects in a data pool. Thus, providing individual responses, along with the composite response, when examining the pattern of neuromuscular responses during a fatiguing task may be warranted.

Previous studies have utilized isokinetic ${ }^{5,6,9}$ or isometric ${ }^{8}$ bilateral muscle actions to examine the individual and composite neuromuscular patterns of responses during fatiguing exercise. However, resistance training is more commonly prescribed using DCER exercises that include both concentric and eccentric portions of the muscle action. In addition, the isokinetic muscle actions required the subjects to elicit maximal force production with each repetition ${ }^{5,6,9}$ while DCER muscle actions at moderate loads require submaximal force production at the start of the fatiguing task. As such, this may lead to differences in the primary factor of fatigue ${ }^{10}$ and show as differences in the neuromuscular responses to fatigue between the different modalities. Thus, while the current literature provides insight on the variability of individual responses compared to the composite data for both isokinetic and isometric muscle actions, these exercises may not reflect what is occurring during more commonly utilized DCER muscle actions. Further, the potential for limb imbalances in the response to fatigue may have implications for training, recovery, and therapy for coaches, practitioners, and clinicians. Specifically, examining the pattern of responses to fatiguing, moderate load resistance exercise may help coaches, practitioners, and clinicians provide recommendations to their athletes and clients for improvement of athletic performance or activities of daily living. Therefore, the purpose of this study was to examine the inter- and intra-individual EMG AMP, EMG MPF, MMG AMP, and MMG MPF patterns of responses of the right and left limbs during DCER, bilateral, leg extension exercise performed to failure at a moderate $(70 \%$ onerepetition maximum [1RM]) load. Based on previous studies, we hypothesized the composite neuromuscular responses between limbs would be similar in both direction and pattern, but there would be large variability in the pattern of neuromuscular responses when comparing the individual limb responses to the composite limb responses as well as the right and left limb responses for each individual.

\section{Scientific Methods}

Participants

Eleven recreationally active men completed this study (Age: $23 \pm 3$ years; height: $175.7 \pm 7.2 \mathrm{~cm}$; body mass: $79.9 \pm 8.7$ $\mathrm{kg})$. The subjects were eligible to participate in this study if they had been consistently participating in a resistance training program for the past year ( $\geq 3$ days per week) and were free from any cardiovascular, metabolic, pulmonary, or neuromuscular diseases or injuries. These subjects were from a large data set that included multiple independent and dependent variables. However, there was no overlap in primary independent or dependent variables in this study and those previously published. ${ }^{11}$ This study was approved by the University Institutional Review Board for Human Subjects (IRB\# 45933). Prior to testing, all the subjects signed an informed consent document and health history questionnaire. 
Protocol

This study consisted of two visits to the exercise physiology laboratory separated by 48 hours. On visit one, the subjects completed anthropometric (height and body mass) measures, followed by the completion of a 1RM for the leg extension exercise. On visit 2 , the subjects completed leg extension repetitions to failure at $70 \% 1 \mathrm{RM}$. During all visits, the EMG and MMG AMP and MPF responses were measured from the vastus lateralis of the right and left limbs.

One-repetition Maximum Testing

During the first visit, the subjects completed a 1RM for the leg extension (Body-Solid, GLCE365, Forest Park, IL, USA). The $1 \mathrm{RM}$ was used to determine the $70 \%$ loading for the subsequent visit. To determine the $1 \mathrm{RM}$, the subjects received three warm-up sets of increasing repetitions and load (8-10 reps, 5-6 reps, and 2-3 reps). On the fourth set, the subjects were given 5 attempts to establish a $1 \mathrm{RM} .{ }^{12} \mathrm{~A} 1 \mathrm{RM}$ was considered successful if full range of motion of the leg extension was achieved. Full range of motion was determined by measuring the distance from the bottom of the ankle when the leg was fully extended $\left(180^{\circ}\right)$ to the ground. This height was recorded and adjusted on a mobile stadiometer for the investigator to visually inspect achievement of the limbs traveling from $90^{\circ} \mathrm{knee}$ bend to $180^{\circ}$ full extension. The subjects received three minutes rest between each warm-up set and between 1RM attempts.

Repetitions Performed to Failure at 70\% 1RM

During the second visit, the subjects completed leg extension repetitions to failure at 70\% 1RM. Prior to completing the repetitions to failure, the subjects completed the same warm-up as previously described for the $1 \mathrm{RM}$ visit to get within 5-10 kg of the working load. The subjects performed the repetitions to failure to a metronome set to 55 beats per minute. This corresponded to $1.1 \mathrm{sec}$ for the concentric phase (leg extended to $180^{\circ}$ ) and $1.1 \mathrm{sec}$ for the eccentric phase (leg returns to starting position of $90^{\circ}$ knee bend). Failure was defined as the inability to complete the repetitions to the set cadence or to perform the repetition through the full range of motion (as described for the 1RM attempt).

Electromyographic and Mechanomyographic Measurements

During the performance of repetitions to failure at 70\% 1RM, a bipolar surface EMG electrode (Ag/AgCL, AccuSensor, Lynn Medical, Wixom, MI, USA) arrangement (30 mm interelectrode distance) was placed on the vastus lateralis of both the right and left thighs. Prior to electrode placement, the skin for each electrode site was shaved, carefully abraded, and cleaned with isopropyl alcohol. The EMG electrodes were placed in accordance with the SENIAM guidelines at $66 \%$ the distance between the lateral superior border of the patella and the anterior superior iliac crest. ${ }^{13}$ In addition, the electrode-placement site was located $5 \mathrm{~cm}$ lateral to the reference line so that the electrodes were over the vastus lateralis muscle. ${ }^{14}$ A goniometer was used to place the electrode at a $20^{\circ}$ angle to approximate the pennation angle of the muscle fibers for the vastus lateralis. ${ }^{15} \mathrm{~A}$ reference electrode was placed over the anterior superior iliac crest of the right limb. The MMG signals were recorded simultaneously with the EMG signals using an accelerometer (Entran EGAS FT, 10, bandwidth 0-200 Hz, dimensions: 1.0 x 1.0 x $0.5 \mathrm{~cm}$, mass 1.0 g sensitivity 10 $\mathrm{mV} \mathrm{g}^{-1}$ ) that was placed between the bipolar electrode arrangement using double-sided adhesive tape. The signal was analyzed from the sensitive axis of the accelerometer positioned perpendicular to the skin surface. The other sensitive axis was positioned along the longitudinal axis of the muscle.

Signal Processing

The raw EMG and MMG signals were sampled at $1 \mathrm{kHz}$ with a 16-bit analog-to-digital converter (Model MP150, BIOPAC Systems, Inc., Santa Barbara, CA, USA). The EMG signals were differentially amplified (EMG 100, BIOPAC Systems, Inc., Santa Barbara, CA, USA; bandwidth = 10-500 Hz; gain: x1,000) and the raw MMG signals were amplified with an in-line amplifier (gain: 200). The signals were recorded and stored in a personal computer for subsequent offline analysis and processing using a custom program written with LabVIEW programming software (version 17.0, National Instruments, Austin, TX). The EMG and MMG signals were zero-meaned and digitally bandpass filtered (fourth-order Butterworth) at 10-500 Hz and 5-100 Hz, respectively. For the MPF analyses, each data segment was processed with a Hamming window and a discrete Fourier transform (DFT) algorithm in accordance with the recommendations of Hermens et al. ${ }^{13}$ The MPF was selected to represent the power spectrum based on the recommendations of Hermens et $a .^{13}$ and was calculated as described by Kwatny et al. ${ }^{16}$ The EMG (microvolts root mean square, $\mu \mathrm{Vrms}$ ) and MMG (root mean square; $\mathrm{m} \cdot \mathrm{s}^{-2}$ ) amplitude (AMP) and frequency (MPF; Hz) values from the middle $1 / 3$ of the concentric portion of the leg extension repetition (leg extends to $180^{\circ}$ ) were calculated for every $10 \%$ of the total repetitions completed during the performance of repetitions to failure at $70 \% 1 \mathrm{RM}$ for the both the right and left vastus lateralis. The EMG AMP, EMG MPF, MMG AMP, and MMG MPF were normalized to the initial 
repetition performed during the $70 \% 1 \mathrm{RM}$ repetitions completed to failure, and every $10 \%$ of repetitions was used for analysis.

\section{Statistical Analysis}

Analyses were performed on the individual and composite (defined as the mean of all subjects) EMG and MMG, AMP and MPF responses for both the right and left vastus lateralis during the performance of leg extension repetitions to failure at $70 \% 1 \mathrm{RM}$. The neuromuscular responses were normalized as a percent of the initial repetition performed to observe the pattern of responses over time, and repetitions were normalized as a percentage of the total repetitions completed to account for differences in the number of repetitions completed to failure among the subjects. Eleven data points were used in the analyses (repetition 1, 10, 20, 30, 40, 50, 60, 70, 80, 90, and 100\% of total repetitions completed). Polynomial regression analyses were used to determine the individual and composite, normalized EMG and MMG, AMP and MPF responses (linear, quadratic, or cubic) vs. percent of total repetitions completed (10-100\% of total repetitions) for both the right and left vastus lateralis muscles. The statistical significance $(\mathrm{p} \leq 0.05)$ for the increment in the proportion of the variance that was accounted for by a higher-degree polynomial was determined using the $F$ test described by Pedhazur. ${ }^{17}$ All analyses were conducted using the Statistical Package for the Social Sciences software (v.24.0. IBM SPSS Inc., Chicago, IL).

\section{Results}

Table 1 includes the composite and individual 1RM values, as well as the load ( $\mathrm{kg}$ ) corresponding to $70 \% 1 \mathrm{RM}$ and the number of repetitions completed at $70 \% 1 \mathrm{RM}$.

\section{EMG Responses}

The polynomial regression analyses for the composite (Figure 1) and individual (Figure 2) EMG AMP and EMG MPF responses for the right and left limbs are presented in Table 2. For the composite EMG AMP, there was a positive, quadratic relationship for both the right $\operatorname{limb}\left(\mathrm{R}=0.992, \mathrm{r}^{2}=0.983, \mathrm{p}<0.001\right)$ and left $\operatorname{limb}\left(\mathrm{R}=0.984, \mathrm{r}^{2}=0.968\right.$, $\mathrm{p}=0.044)$. For the right limb, 3 of the 11 subjects also demonstrated a positive, quadratic relationship $\left(\mathrm{r}^{2}=0.602-\right.$ 0.777), while 2 subjects presented a positive, linear relationship $\left(\mathrm{r}^{2}=0.642-0.764\right), 1$ subject demonstrated a positive, cubic relationship $\left(\mathrm{r}^{2}=0.908\right)$, and 5 subjects exhibited no significant relationship. For the left limb, 2 of the 11 subjects demonstrated a positive, quadratic relationship $\left(r^{2}=0.564-0.793\right)$, consistent with that demonstrated for the composite, left limb EMG AMP response. However, 4 subjects demonstrated a positive, linear relationship $\left(\mathrm{r}^{2}=0.367\right.$ - 0.791), 1 subject demonstrated a positive, cubic relationship $\left(r^{2}=0.952\right)$, and 4 subjects exhibited no significant relationship.

Table 1. Individual and composite one-repetition maximum (1RM) values, the load corresponding to $70 \% 1 \mathrm{RM}$, and the number of repetitions completed at $70 \% 1 \mathrm{RM}$.

\begin{tabular}{cccc}
\hline Subject & $\mathbf{1 R M}(\mathbf{k g})$ & $\mathbf{7 0 \%} \mathbf{1 R M}(\mathbf{k g})$ & $\begin{array}{c}\text { Repetitions } \\
\text { completed } \boldsymbol{a t} \\
\mathbf{7 0 \%} \mathbf{1 R M}\end{array}$ \\
\hline 1 & 75 & 53 & 13 \\
2 & 66 & 46 & 18 \\
3 & 105 & 74 & 12 \\
4 & 111 & 78 & 14 \\
5 & 102 & 71 & 15 \\
6 & 82 & 57 & 14 \\
7 & 73 & 51 & 15 \\
8 & 100 & 70 & 18 \\
9 & 82 & 57 & 14 \\
10 & 98 & 69 & 15 \\
11 & 66 & 46 & $\mathbf{1 5} \pm \mathbf{2}$ \\
\hline Composite & $\mathbf{8 7 \pm 1 6}$ & $\mathbf{6 1} \pm \mathbf{1 1}$ &
\end{tabular}

For the EMG MPF responses, the composite data for the right limb demonstrated a negative, cubic relationship $(\mathrm{R}=$ $\left.-0.982, \mathrm{r}^{2}=0.964, \mathrm{p}=0.001\right)$. For the individual responses, 2 of the 11 subjects also demonstrated a negative, cubic relationship $\left(\mathrm{r}^{2}=0.826-0.853\right)$ for the right limb and 1 subject demonstrated a positive, cubic relationship $\left(\mathrm{r}^{2}=\right.$ 0.802). Three subjects demonstrated a negative, linear relationship $\left(\mathrm{r}^{2}=0.497-0.799\right)$ and 5 subjects exhibited no 
significant relationship. For the left limb, the composite data demonstrated a negative, quadratic relationship $(\mathrm{R}=$ $\left.0.950, \mathrm{r}^{2}=0.903, \mathrm{p}=0.008\right)$ for EMG MPF. Only 1 of the 11 subjects also demonstrated a negative, quadratic relationship $\left(r^{2}=0.944\right)$, while 5 of the 11 subjects demonstrated a negative, linear relationship $\left(r^{2}=0.462-0.815\right), 1$ subject demonstrated a positive, cubic relationship $\left(r^{2}=0.642\right)$, and 4 subjects demonstrated no significant relationship.

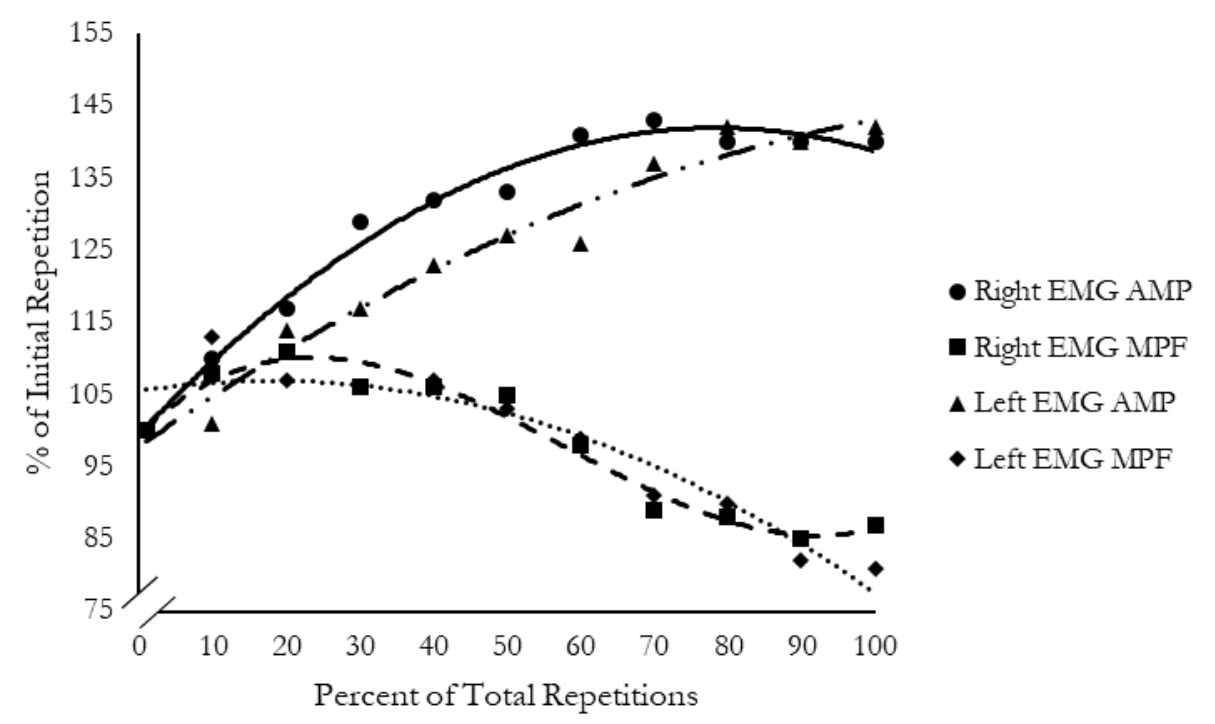

Figure 1. Polynomial regression analyses (linear, quadratic, or cubic) for the composite electromyographic (EMG) amplitude (AMP) and mean power frequency (MPF) responses for the right and left vastus lateralis. All EMG responses demonstrated significant relationships. See Table 2 for polynomial regression analyses. 
Table 2. Model fit, correlation, and p-value for the polynomial regression analyses for the electromyography (EMG) amplitude (AMP) and mean power frequency (MPF) responses for the right and left vastus lateralis.

\begin{tabular}{|c|c|c|c|c|c|c|c|c|c|c|c|c|}
\hline \multirow[t]{2}{*}{ Subject } & \multicolumn{3}{|c|}{ Right EMG AMP } & \multicolumn{3}{|c|}{ Left EMG AMP } & \multicolumn{3}{|c|}{ Right EMG MPF } & \multicolumn{3}{|c|}{ Left EMG MPF } \\
\hline & Model & Correlation & $\begin{array}{l}p- \\
\text { value }\end{array}$ & Model & Correlation & $\begin{array}{l}p- \\
\text { value }\end{array}$ & Model & Correlation & $p$-value & Model & Correlation & $\begin{array}{l}p- \\
\text { value }\end{array}$ \\
\hline 1 & None & - & - & None & - & - & Linear & -0.874 & $<0.001$ & Linear & -0.724 & 0.012 \\
\hline 2 & None & - & - & None & - & - & None & - & - & None & - & - \\
\hline 3 & Cubic & 0.953 & 0.007 & Linear & 0.671 & 0.024 & Cubic & -0.909 & 0.029 & None & - & - \\
\hline 4 & None & - & - & Linear & 0.606 & 0.048 & None & - & - & Linear & -0.849 & 0.001 \\
\hline 5 & Quadratic & 0.776 & 0.037 & Linear & 0.889 & $<0.001$ & Cubic & -0.853 & 0.046 & Linear & -0.679 & 0.021 \\
\hline 6 & Linear & 0.801 & 0.003 & Quadratic & 0.890 & 0.035 & None & - & - & None & - & - \\
\hline 7 & Linear & 0.874 & $<0.001$ & Cubic & 0.976 & 0.029 & None & - & - & None & - & - \\
\hline 8 & None & - & - & None & - & - & Cubic & 0.896 & 0.009 & Linear & -0.770 & 0.006 \\
\hline 9 & Quadratic & 0.881 & 0.048 & Linear & 0.833 & 0.001 & None & - & - & Quadratic & -0.971 & 0.005 \\
\hline 10 & Quadratic & 0.785 & 0.010 & Quadratic & 0.751 & 0.012 & Linear & -0.705 & 0.015 & Cubic & 0.801 & 0.049 \\
\hline 11 & None & - & - & None & - & - & Linear & -0.894 & $<0.001$ & Linear & -0.903 & $<0.001$ \\
\hline Composite & Quadratic & 0.992 & $<0.001$ & Quadratic & 0.984 & 0.044 & Cubic & -0.982 & 0.001 & Quadratic & -0.950 & 0.008 \\
\hline
\end{tabular}



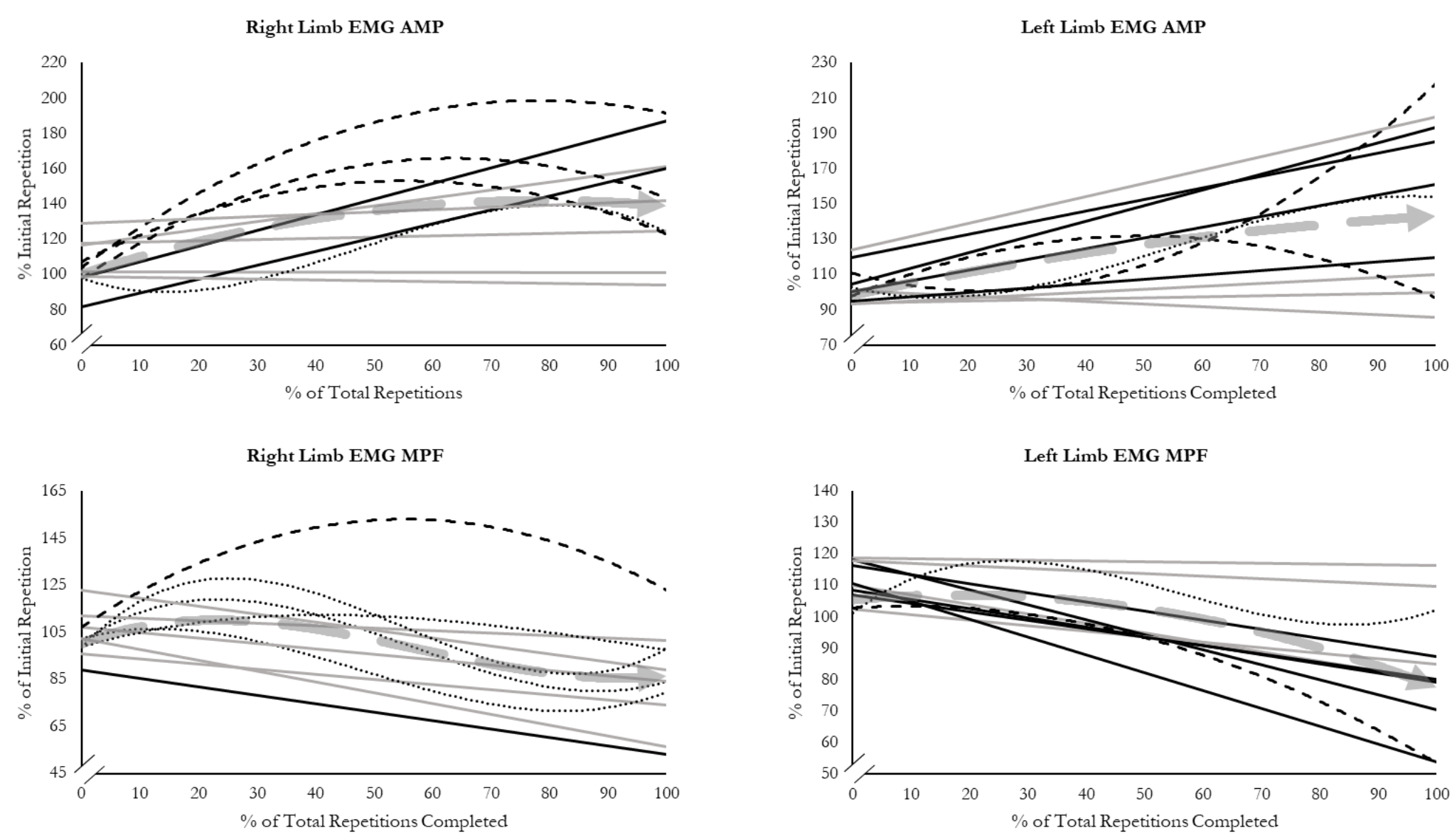

Figure 2. The individual electromyographic (EMG) amplitude (AMP) and mean power frequency (MPF) responses for the right and left limbs. A solid line reflects a linear relationship, a dashed line represents a quadratic relationship, and a dotted line represents a cubic relationship. The light gray solid lines represent individual subjects who demonstrated no significant relationship. The light gray dashed line represents the composite relationship. See Table 2 for individual and composite polynomial regression analyses. 


\section{MMG Responses}

The polynomial regression analyses for the composite (Figure 3) and individual (Figure 4) MMG AMP and MMG MPF responses for the right and left limbs are presented in Table 3. For the right limb, the MMG AMP composite data demonstrated a positive, quadratic relationship $\left(\mathrm{R}=0.982, \mathrm{r}^{2}=0.965, \mathrm{p}=0.004\right)$, while the MMG AMP composite data for the left limb demonstrated a positive, linear relationship $\left(r=0.942, \mathrm{r}^{2}=0.888, \mathrm{p}<0.001\right)$. For the right limb, 3 of the 11 subjects demonstrated a positive, quadratic relationship $\left(r^{2}=0.593-0.890\right), 1$ subject demonstrated a positive, linear relationship $\left(\mathrm{r}^{2}=0.544\right), 1$ subject demonstrated a positive, cubic relationship $\left(\mathrm{r}^{2}=0.872\right)$, and 6 subjects demonstrated no significant relationship. For the left limb, 5 of the 11 subjects demonstrated a positive, linear relationship $\left(r^{2}=0.401-0.440\right)$ consistent with the composite results, while the remaining 6 subjects demonstrated no significant relationship.

The composite data for MMG MPF demonstrated a negative, linear relationship for both the right $\left(r=-0.842, \mathrm{r}^{2}\right.$ $=0.708, \mathrm{p}=0.001)$ and the left limbs $\left(\mathrm{r}=-0.901, \mathrm{r}^{2}=0.811, \mathrm{p}<0.001\right)$. For the right limb, 3 of the 11 subjects also demonstrated a negative, linear relationship $\left(\mathrm{r}^{2}=0.559-0.849\right), 1$ subject demonstrated a negative, quadratic relationship $\left(\mathrm{r}^{2}=0.681\right), 1$ subject demonstrated a positive, cubic relationship $\left(\mathrm{r}^{2}=0.645\right)$, and 2 subjects demonstrated a negative, cubic relationship $\left(r^{2}=0.541-0.927\right)$, while 4 subjects exhibited no significant relationship. For the left limb, 4 of the 11 subjects demonstrated a negative, linear relationship consistent with the composite data for the left limb MMG MPF, while 1 subject demonstrated a negative, quadratic relationship, and 6 subjects exhibited no significant relationship.

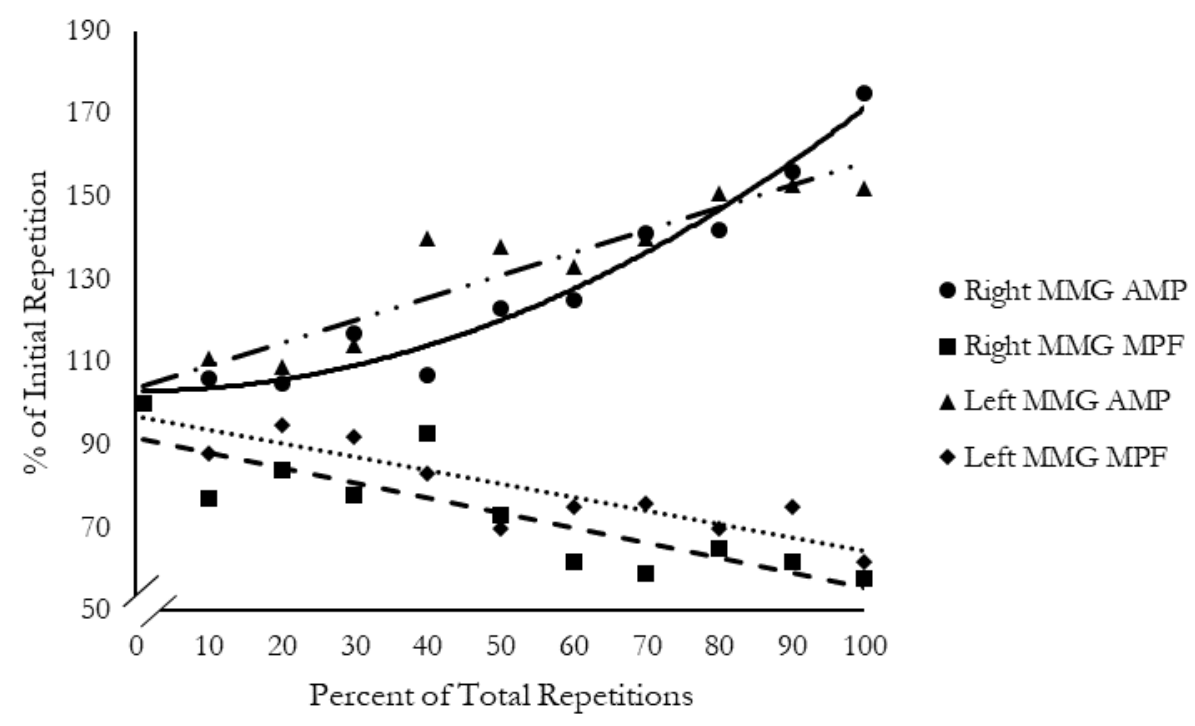

Figure 3. Polynomial regression analyses (linear, quadratic, or cubic) for the composite mechanomyographic (MMG) amplitude (AMP) and mean power frequency (MPF) responses for the right and left vastus lateralis. All MMG responses demonstrated significant relationships. See Table 3 for polynomial regression analyses. 
and PERFORMANCE

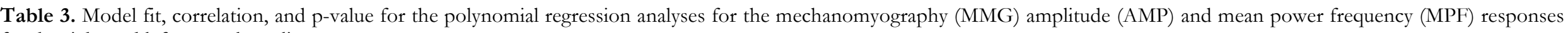
for the right and left vastus lateralis.

\begin{tabular}{|c|c|c|c|c|c|c|c|c|c|c|c|c|}
\hline \multirow[t]{2}{*}{ Subject } & \multicolumn{3}{|c|}{ Right MMG AMP } & \multicolumn{3}{|c|}{ Left MMG AMP } & \multicolumn{3}{|c|}{ Right MMG MPF } & \multicolumn{3}{|c|}{ Left MMG MPF } \\
\hline & Model & Correlation & $\begin{array}{l}\mathrm{p}- \\
\text { value }\end{array}$ & Model & Correlation & $\begin{array}{l}\mathrm{p}- \\
\text { value }\end{array}$ & Model & Correlation & $\begin{array}{l}\mathrm{p}- \\
\text { value }\end{array}$ & Model & Correlation & $\begin{array}{l}\mathrm{p}- \\
\text { value }\end{array}$ \\
\hline 1 & None & - & - & Linear & 0.633 & 0.036 & Linear & -0.748 & 0.008 & Linear & -0.625 & 0.040 \\
\hline 2 & Quadratic & 0.770 & 0.026 & None & - & - & Quadratic & -0.825 & 0.009 & None & - & - \\
\hline 3 & None & - & - & Linear & 0.664 & 0.026 & Linear & -0.921 & $<0.001$ & None & - & - \\
\hline 4 & Quadratic & 0.943 & 0.007 & None & - & - & Linear & -0.794 & 0.004 & Linear & -0.744 & 0.009 \\
\hline 5 & Linear & 0.737 & 0.010 & Linear & 0.640 & 0.034 & None & - & - & None & - & - \\
\hline 6 & None & - & - & None & - & - & Cubic & 0.803 & 0.038 & Quadratic & -0.776 & 0.019 \\
\hline 7 & None & - & - & None & - & - & None & - & - & None & - & - \\
\hline 8 & None & - & - & None & - & - & None & - & - & Linear & -0.850 & 0.001 \\
\hline 9 & Cubic & 0.934 & 0.013 & Linear & 0.692 & 0.018 & Cubic & -0.963 & 0.006 & Linear & -0.913 & $<0.001$ \\
\hline 11 & None & - & - & None & - & - & Cubic & -0.736 & 0.025 & None & - & - \\
\hline Composite & Quadratic & 0.982 & 0.004 & Linear & 0.942 & $<0.001$ & Linear & -0.842 & 0.001 & Linear & -0.901 & $<0.001$ \\
\hline
\end{tabular}


Right Limb MMG AMP

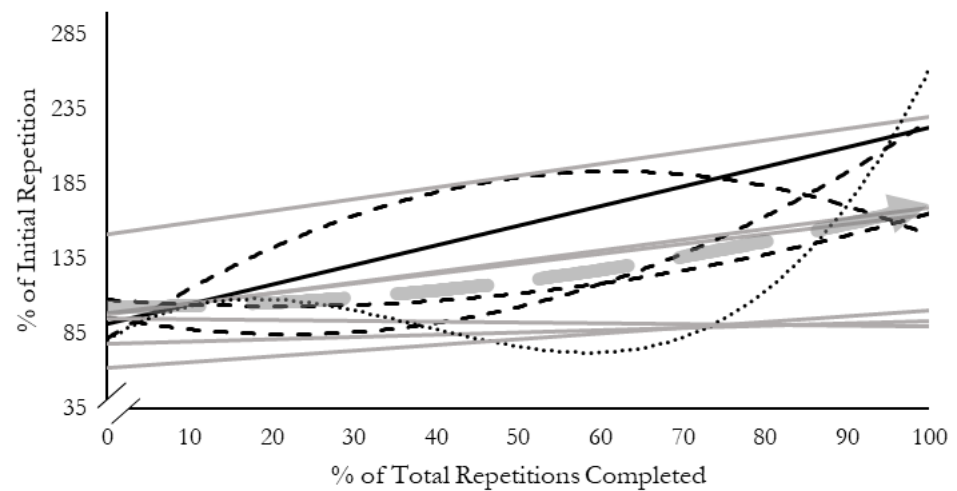

Right Limb MMG MPF

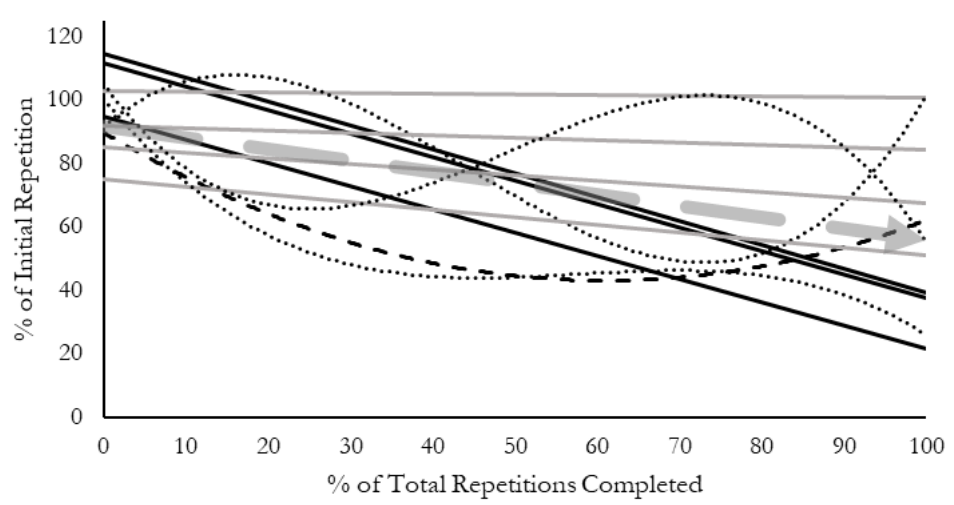

Left Limb MMG AMP

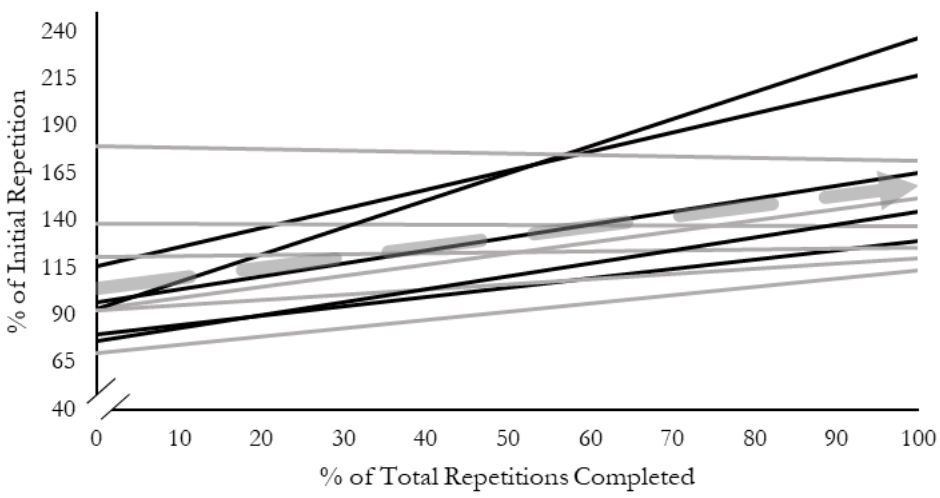

Left Limb MMG MPF

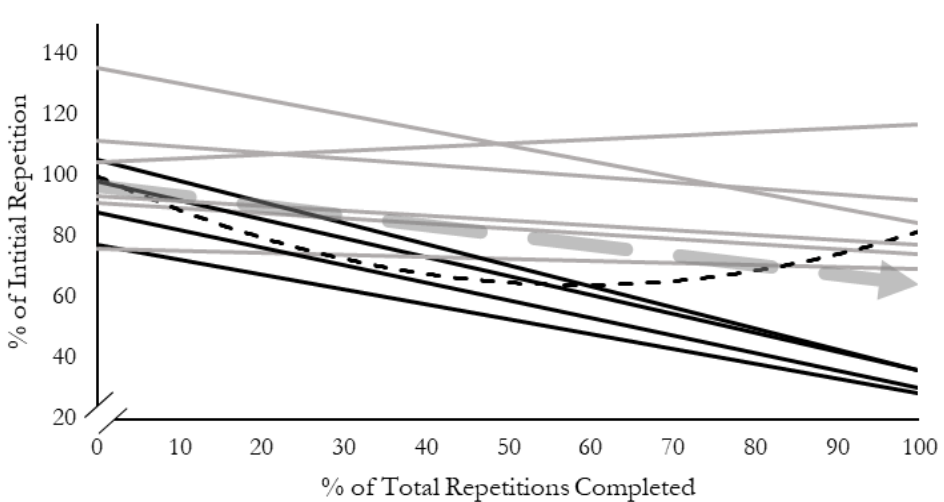

Figure 4. The individual mechanomyographic (MMG) amplitude (AMP) and mean power frequency (MPF) responses for the right and left limbs. A solid line reflects a linear relationship, a dashed line represents a quadratic relationship, and a dotted line represents a cubic relationship. The light gray solid lines represent individual subjects who demonstrated no significant relationship. The light gray dashed line represents the composite relationship. See Table 3 for individual and composite polynomial regression analyses. 


\begin{abstract}
Discussion
Composite Responses

In the current study, the subjects completed $15 \pm 2$ repetitions of the bilateral, DCER, leg extension exercise performed at $70 \% 1 \mathrm{RM}(61 \pm 11 \mathrm{~kg})$. For EMG AMP and MMG MPF, both the right and left limbs demonstrated the same direction and pattern of responses. For EMG MPF and MMG AMP, the right and left limbs demonstrated the same direction of response, but different patterns of response (Figures $1 \& 3$ ). Thus, $100 \%$ of the signals were consistent in direction (i.e., positive or negative) between right and left limbs, and 50\% of the signals (EMG AMP and MMG MPF) demonstrated the same pattern and direction of response between the right and left limbs during fatiguing, bilateral, DCER, leg extensions performed at $70 \% 1 \mathrm{RM}$. This is consistent with previous investigations that have examined the interlimb responses during maximal, bilateral, isokinetic muscle actions of the leg extensors performed at $180^{\circ} \mathrm{s}^{-1}, 6,8,9$ maximal, bilateral, isometric muscle actions of the leg extensors, ${ }^{18}$ and submaximal, bilateral, isometric muscle actions of the leg extensors performed at $20 \%$ maximal voluntary contraction, ${ }^{19}$ and reported no difference in the neuromuscular responses between limbs. Therefore, the consistency in the composite response between limbs during bilateral muscle actions can be extended to traditional DCER exercise typically prescribed during resistance training protocols.
\end{abstract}

Previously, the common neuromuscular responses observed between contralateral limbs have been attributed to common drive that is apparent between two homologous muscles at rest. ${ }^{20,21}$ During maximal bilateral muscle actions, Howard and Enoka 22 identified the ability to maximally activate the contralateral limbs as "neural integration" which enhances bilateral facilitation (i.e., the ability of the right and left limbs to produce more force than the sum of unilateral left plus unilateral right limb force). Currently, there is conflicting evidence regarding coherence between limbs during fatiguing tasks. ${ }^{20,21,23,24}$ Specifically, Oda and Moritani ${ }^{20}$ demonstrated high cross-correlation between the biceps brachii muscle of the right and left limbs at the start of a fatiguing, maximal, bilateral muscle action that quickly decreased during the fatiguing 1-minute contraction. More recently, Boonstra et al. ${ }^{23}$ demonstrated similar interlimb coherence between the right and left triceps during fatiguing forearm extension sustained at $90^{\circ}$ forearm flexion at $20 \%$ and $40 \%$ maximal voluntary contraction. To note, authors suggested the interlimb coherence may have arisen from common afferent feedback that can influence motor unit discharge rate. ${ }^{23}$ Further, in contrast to previous work, ${ }^{20,21}$ authors indicated an association between fatigue and coherence, such that coherence increased over time throughout fatiguing trials during isometric muscle actions of the leg extensors ${ }^{24}$ and isometric muscle actions of the forearm extensors. ${ }^{23}$ For both upper- and lower-limb bilateral muscle actions, increases in coherence also accompanied increases in EMG AMP and decreases in EMG median power frequency, indicating the increase in coherence was likely due to increased motor unit synchronization between the right and left limbs. ${ }^{23,24}$ Authors have hypothesized interlimb coherence and motor unit synchronization may arise from "neural crosstalk" in different areas of the brain. 21,23,24 Thus, it appears during bilateral muscle actions, the similar neuromuscular responses reported between contralateral limbs may be due to increased neural crosstalk and, consequently, motor unit synchronization to couple the mirror movements between limbs and allow for continued force development of both limbs. Collectively, the consistency of neuromuscular responses between the right and left limbs in the current study agree with previous studies examining neuromuscular responses in contralateral limbs during isokinetic and isometric muscle actions and extends the hypothesis of common drive to include bilateral, DCER muscle actions.

Inter-Individual Responses

Collectively, for the EMG and MMG signals for both the right and the left limbs, less than half of the subjects (18$45 \%$ ) demonstrated the same response as the composite data, while $9-54 \%$ of the individual subjects demonstrated either a different response than the composite or demonstrated no relationship (Figures $2 \& 4)$. This is consistent with individual responses previously reported during maximal, bilateral, isokinetic muscle actions of the leg extensors performed at $180^{\circ} \mathrm{s}^{-1} .6$,9 Specifically, Anders et al. ${ }^{6}$ reported that $8-85 \%$ of the individuals demonstrated the same relationship as the composite response for the EMG and MMG signals. To corroborate this, Neltner and colleagues ${ }^{9}$ reported an average of $31 \%$ of the subjects demonstrated the same pattern of neuromuscular responses for the right and left limbs as the composite response. Further, during maximal, bilateral, isometric muscle actions of the leg extensors performed at a force corresponding to a rating of perceived exertion (RPE) $=1, \mathrm{RPE}=5$, and $\mathrm{RPE}=8$, Keller et al. ${ }^{8}$ reported consistency between individual limb neuromuscular responses and the composite neuromuscular responses for $25-92 \%$ of individuals, $8-92 \%$ of individuals, and $0-33 \%$ of individuals, respectively. In the current study, 
the average consistency in neuromuscular responses for the individuals compared to the composite response was $26 \%$. It is important to note that the current study employed bilateral, DCER muscle actions of the leg extensors which were completed until failure. Failure in the current study was defined as the inability to complete the leg extension repetition through the full range of motion or the inability to maintain the desired repetition cadence. Previous studies had the subjects complete maximal, bilateral, isokinetic muscle actions of the leg extensors performed at a rate of $180^{\circ} \mathrm{s}^{-1}$, and defined failure as a decline in torque production to a value that was less than $50 \%$ peak torque ${ }^{6}$ or a decline in torque production to a value that was less than $30 \%$ peak torque. ${ }^{9}$ Further, Keller et al. ${ }^{8}$ anchored intensity based on a force corresponding to a RPE, which could vary greatly between individual subjects. Specifically, individual force values ranged from $4.8 \%$ to $15.2 \%$ maximal voluntary contraction for RPE $=1,25.1 \%$ to $60.6 \%$ maximal voluntary contraction for $\mathrm{RPE}=5$, and $44.7 \%$ to $89.6 \%$ maximal voluntary contraction for RPE $=8 .{ }^{8}$ Therefore, the lower percent of consistency between individual neuromuscular responses and the composite neuromuscular responses in the current study compared to previous studies may be due to a different modality and load lifted, as well as the definition of task failure and the potential for extraneous variables to affect the fatigue response. To this point, DCER muscle actions allow for shifts in limb and foot position during repetitions, which can influence the EMG signal and may vary greatly between individuals. ${ }^{25,26}$ In addition, maximal (i.e., 100\% 1RM) muscle actions and submaximal, moderate-load (i.e., 80\% 1RM) DCER muscle actions may have different primary sources of fatigue. ${ }^{10}$ Specifically, authors have speculated during maximal muscle actions, fatigue may be primarily due to the inability to drive the agonist muscle groups, while fatigue during submaximal muscle actions may be primarily due to peripheral factors such as metabolite accumulation. ${ }^{10}$ Thus, depending on the point when the subjects were required to recruit near maximal levels of motor units to continue the performance of repetitions to failure at $70 \% 1 \mathrm{RM}$ in the current study, the neuromuscular responses and primary source of fatigue may vary between individuals. Based on these findings, this indicates composite, neuromuscular responses during fatiguing tasks may not be reflective of the individual's neuromuscular response to fatiguing exercise. Thus, this study supports previous recommendations ${ }^{6,9}$ that suggest both individual and composite responses should be reported when examining neuromuscular parameters during fatiguing muscle actions. Further, this indicates there may be variability in the primary source of fatigue during isokinetic muscle actions and DCER muscle actions that lead to inconsistencies in the neuromuscular responses between the two modalities.

\section{Intra-Individual Responses}

In the current study, only $14 \%$ of the signals contained individuals who demonstrated the same pattern and direction of response for both limbs and $20 \%$ of the signals contained individuals whose right and left limbs demonstrated the same direction (positive or negative) of response, but exhibited a different pattern, for both limbs (Tables 2 \& 3 ). Moreover, $32 \%$ of the signals had individuals who demonstrated no relationship for both the right and left limbs and the remaining $34 \%$ of the signals contained individuals who demonstrated a combination of different patterns, directions, or no relationship for the neuromuscular responses of the right and left limbs (Tables $2 \& 3$ ). Thus, most of the individual subjects demonstrated variability in the responses between the right or left limbs or exhibited no relationship for either limb. Consistent with the current study, previous studies examining intra-limb responses during maximal, bilateral, isokinetic muscle actions of the leg extensors reported 19-22\% of the signals contained individuals whose right and left limbs resulted in the same pattern and direction of neuromuscular responses, while only 4-7\% of the signals contained individuals who demonstrated the same direction (positive or negative) but a different pattern of responses between right and left.6,9 In addition, 37-46\% of the signals contained individuals who demonstrated no relationship for both the right and left limbs, and $25-40 \%$ of the signals contained individuals who demonstrated a combination of different directions, patterns, or no relationship for the right and left limbs. ${ }^{6,9}$ Further, when anchored to a force corresponding to an RPE $=1,8-83 \%$ of the signals contained individuals who demonstrated the same response between dominant and nondominant limbs, while the remaining $17-92 \%$ of the signals differed in pattern or direction or had no relationship for the neuromuscular signals. ${ }^{8}$ At higher forces, such as that anchored to an RPE $=$ 5 or $\mathrm{RPE}=8$, the consistency in neuromuscular responses between limbs decreased and ranged from $0-58 \%$ of the signals that contained individuals with the same response for the dominant and nondominant limbs. ${ }^{8}$ Conversely, most of the signals at higher force values contained individuals who demonstrated no relationship for either limb, or a combination of directions, patterns, and no relationships between the dominant and nondominant limbs. ${ }^{8}$ Thus, the force produced may influence the consistency of responses between limbs within an individual subject. ${ }^{8}$ This provides further evidence that composite responses do not fully reflect the neuromuscular activity occurring at the intraindividual level. In addition, this may indicate there is variability between limbs in motor unit activation strategies utilized to maintain force production during fatiguing tasks. Specifically, only examining the neuromuscular responses of one limb may not fully explain the fatigue response occurring in the contralateral limb during bilateral leg extension exercise. This may lead to inappropriate training practices or therapy recommendations if single-limb function is 
essential to the athlete or patient being examined, such as in triple jump athletes or firefighters who may consistently use the same limb when stepping on or off the fire truck.

\section{Conclusions}

For the composite responses, $100 \%$ of the signals demonstrated the same direction of response between the right and left limbs, and $50 \%$ of the signals demonstrated the same pattern and direction between the right and left limbs. In general, half of the subjects in the current study demonstrated the same neuromuscular responses in the right and left limbs as the right and left limb composite responses. Although the composite responses allude to a common drive between limbs that aids in force production and mirror movement during bilateral muscle actions, ${ }^{20,21,23,24}$ there was considerable inter- and intra-individual variability in responses. Specifically, only $33 \%$ of the subjects demonstrated the same neuromuscular responses in both the right and left limbs, while $66 \%$ of the subjects either demonstrated no relationship in both the right and left limbs, or a combination of no relationship and different patterns or directions of responses in the right and left limbs. This highlights the importance of providing both individual and composite neuromuscular responses when examining fatigue as the composite response may not fully reflect the true response occurring at the individual level. This information may help coaches, practitioners, and clinicians identify potential inconsistencies or imbalances in the fatigue response between the right and left limbs during bilateral muscle actions. This may help provide recommendations for improving limb symmetry, particularly in individuals or athletes who may favor one limb for activities of daily living or athletic performance, respectively.

\section{Acknowledgements: None}

Conflicts of Interest: The authors declare no conflicts of interest.

\section{References}

1. Basmajian JV. Muscle Alive. Baltimore, MD: The Williams \& Wilkins Company; 1979.

2. Beck TW, Housh TJ, Cramer JT, Weir JP, Johnson GO, Coburn JW, Malek MH, Mielke M. Mechanomyoraphic amplitude and frequency responses during dynamic muscle actions: a comprehensive review. BioMedical Engineering OnLine. 2005;4:67.

3. Beck TW, Housh TJ, Johnson GO, Cramer JT, Weir JP, Coburn JW, Malek MH. Does the frequency content of the surface mechanomyographic signal reflect motor unit firing rates? A brief review. Journal of Electromyography and Kinesiology. 2007;17:1-13.

4. Beck TW, Housh TJ. Use of electromyography in studying human movement. Routledge Handbook of Biomechanics and Human Movement Science. 2008;214-230.

5. Anders JPV, Keller JL, Neltner TJ, Housh TJ, Schmidt RJ, Johnson GO. Task-specific performance fatigability and the bilateral deficit during isokinetic leg extensions. J Musculoskelet Neuronal Interact. 2021;21:412.

6. Anders JPV, Smith CM, Keller JL, Hill EC, Housh TJ, Schmidt RJ, Johnson GO. Inter- and intra-individual differences in EMG and MMG during maximal, bilateral, dynamic leg extensions. Sports. 2019;7:175.

7. Dinyer TK, Byrd MT, Succi PJ, Bergstrom HC. The time course of changes in neuromuscular responses during the performance of leg extension repetitions to failure below and above critical resistance in women. J Strength Cond Res. 2020.

8. Keller JL, Housh TJ, Anders JPV, Neltner TJ, Schmidt RJ, Johnson GO. Similar performance fatigability and neuromuscular responses following sustained bilateral tasks above and below critical force. Eur J Appl Physiol. 2021;121:1111-1124.

9. Neltner TJ, Housh TJ, Smith CM, Anders JPV, Keller JL, Hill EC, Schmidt RJ, Johnson GO. Similar fatigueinduced changes in neuromuscular patterns of responses for contralateral legs during maximal bilateral leg extensions. JEPonline. 2020;23:1-17.

10. Walker S, Davis L, Avela J, Häkkinen K. Neuromuscular fatigue during dynamic maximal strength and hypertrophic resistance loadings. Journal of Electromyography and Kinesiology. 2012;22:356-362.

11. Dinyer TK, Soucie EP, Succi PJ, Voskuil CC, Byrd MT, Bergstrom HC. Muscular performance and neuromuscular fatigue are not sex-dependent during low-load fatiguing bilateral leg extension exercise. NeuroSports. 2020;1.

12. Sheppard JM, Triplett NT. Program design for resistance training. In: Haff GG, Triplett NT, eds. Essentials of Strength and Conditioning. Champaign, IL: Human Kinetics; 2016:453-458. 
13. Hermens HJ, Freriks B, Merletti R, Stegeman D, Blok J, rau G, Disselhorst-Klug C, Hägg G.European recommendations for surface electromyography. Roessingh Research and Development. 1999;8:13-54.

14. Malek MH, Coburn JW, Weir JP, Beck TW, Housh TJ. The effects of innervation zone on electromyographic amplitude and mean power frequency during incremental cycle ergometry. Journal of Neuroscience Methods. 2006;155:126-133.

15. Abe T, Kumagai K, Brechue WF. Fascicle length of leg muscles is greater in sprinters than in distance runners. Med Sci Sports Exerc. 2000;32:1125-1129.

16. Kwatny E, Thomas DH, Kwatny HG. An application of signal processing techniques to the study of myoelectric signals. IEEE Transactions on Bio-Medical Engineering. 1970;17:303-313.

17. Pedhazur EJ. Curvilinear regression analysis. In: Multiple Regression in behavioral Research: Explanation and Prediction. Orlando, FL: Harcourt College Publishers; 1997:520-521.

18. Matkowski B, Martin A, Lepers R. Comparison of maximal unilateral versus bilateral voluntary contraction force. Eur J Appl Physiol. 2011;111:1571-1578.

19. Matkowski B, Place N, Martin A, Lepers R. Neuromuscular fatigue differs following unilateral vs bilateral sustained submaximal contractions. Scand J Med Sci Sports. 2011;21:268-276.

20. Oda S, Moritani T. Cross-correlation of bilateral differences in fatigue during sustained maximal voluntary contraction. Eur J Appl Physiol. 1995;70:305-310.

21. Oda S. Motor control for bilateral muscular contractions in humans. Japanese Journal of Physiology.1997;47:487498.

22. Howard JD, Enoka RM. Maximum bilateral contractions are modified by neurally mediated interlimb effects. J Appl Physiol. 1991;70:306-316.

23. Boonstra TW, Daffertshofer A, van As E, van der Vlugt S, Beek PJ. Bilteral motor unit synchronization is functionally organized. Ex Bran Res. 2007;178:79-88.

24. Boonstra TW, Daffertshofer A, van Ditshuizen JC, van den Heuvel MRC, Hofman C, Willigenburg NW, Beek PJ. Fatigue-related changes in motor-unit synchronization of quadriceps muscles within and across legs. Journal of Electromyography and Kinesiology. 2008;18:717-731.

25. Akima H, Saito A. Inverse activation between the deeper vastus intermedius and superficial muscles in the quadriceps during dynamic knee extension. Muscle Nerve. 2013;47:682-690.

26. Signorile JF, Lew KM, Stoutengerg M, Pluchino A, Lewis JE, Gao J. Range of motion and leg rotation affect electromyography activation levels of the superficial quadriceps muscles during leg extension. J Strength Cond Res. 2014;28:2536-2545. 